\title{
Analysis of Various Techniques for Ovarian Cyst Segmentation
}

\author{
Sheela. S ${ }^{\mathrm{a}, \mathrm{c}, 1}$, Subashini. $\mathrm{V}^{\mathrm{c}}$, M.Sumathi ${ }^{\mathrm{b}}$, Roopsree. $\mathrm{G}^{\mathrm{c}}$, Soundhar.B ${ }^{\mathrm{c}}$, Ramya.R ${ }^{\mathrm{c}}$ \\ ${ }^{a}$ Research Scholar, Sathyabama Institute of Science and Technology \\ ${ }^{b}$ Professor, Sathyabama Institute of Science and Technology \\ ${ }^{c}$ Department of ECE, Rajalakshmi Institute of Technology, India
}

\begin{abstract}
In the female reproductive system, ovary plays a major role. The diseases caused by ovaries are ovarian cysts, ovarian cancer, menstrual cycle disorder and polycystic ovarian syndrome(PCOS). PCOS in women mainly causes infertility. The method of analyzing a polycystic ovarian image varies with every individual patient and a great deal for the image analyst to segment the cyst. This paper reviews several segmentation algorithms like Level set algorithm, K-means clustering and Adaptive thresholding segments the ovarian cyst from the ultrasound images exactly. The four parameters such as accuracy, jaccard coefficient, precision, and sensitivity are used for the evaluation of segmentation processes.
\end{abstract}

Keywords: PCOS, Segmentation, Image processing, Adaptive thresholding, multiple follicles.

\section{Introduction}

In the field of medical science, image processing plays a dynamic role. Polycystic Ovarian Syndrome is a medical disorder that affects the ovary. It appears after the puberty stage in women [1]. If this condition persists for a longer period, it leads to severe complications. Various Imaging modalities like CT, MRI, and Ultrasound imaging are the source tools to diagnose and study the various types of diseases [2]. Ultrasound imaging is the applicable imaging modality for the diagnosis of PCOS, due to its high sensitivity and moderate specificity. Ovarian cyst ultrasound images has to be segmented accurately because it contains multiple follicles combined. Before segmenting the follicles, the ultrasound images are pre-processed for the enhancement and removal of noise [3]. Next, ovarian cysts are segmented using a Level set algorithm, K-means clustering, and Adaptive thresholding method. The performance of these algorithms is evaluated by using accuracy, jaccard coefficient, precision, sensitivity [4]. Finally, this paper ends with the conclusive comment that the best segmentation is achieved using the Adaptive Thresholding technique.

\footnotetext{
${ }^{1}$ S.Sheela, Research Scholar, Sathyabama Institute of Science and Technology, Chennai, India; E-mail: ssheela97@gmail.com
} 


\section{Segmentation}

In medical image processing segmenting an image is a crucial process, as these images will be in digital form and they are separated into various pixels. The characteristics of each pixelcan be categorized based on texture, intensity, colour, regions [5-6]. Many researchers came up with various segmentation algorithms. But the segmentation of ultrasound Polycystic Ovarian images is still a difficult task.

Commonly used segmentation algorithms are discussed below:

2.1 Level set algorithm

$2.2 \mathrm{~K}$-means clustering

2.3 Adaptive thresholding

\subsection{Level Set Algorithm}

Several level sets are generally used in segmentation. It governs the topological change with greater computational ability (Qian \& Weng, 2016). The key idea of this formula is expressed as a set of zero level function, then level determination function is integrated into the expression of evolution of the curve [7]. Finally, the measure of curve evolution is attained by changing its zero level set function.

A point on the curve is always satisfied by the following equation:

$\varphi(x, y, t)=0$

By differentiating this equation with respect to $t$, gives :

$\varphi_{t}+\varphi_{x} \frac{d x}{d t}+\varphi_{y} \frac{d y}{d t}=0$

By the chain rule, the expressions are attained below:

$\varphi_{t}-F|\Delta \varphi|=0$

By partial differential equation it is concluded as follows:

$\varphi(x, y, t)= \pm d$

$\mathrm{F}$ - speed of the curve at every point. $\mathrm{F}$ contains on-board information images $\mathrm{C}$ (X, $\mathrm{Y})$. When the border object is nearer to the curve, $\mathrm{C}(\mathrm{X}, \mathrm{Y})$ makes $\mathrm{F}$ to be zero, and finally, at the edge of the object the curve will be terminated.

The velocity function $\mathrm{F}$ :

$F=C(x, y)\left(F_{0}+F(k)\right)$

$C(x, y)=\frac{1}{1+\left\|\Delta G_{\sigma^{*} I}(x, y)\right\|^{m}}$

$k=\Delta \frac{\Delta \varphi}{|\Delta \varphi|}$

$F_{0}= \pm 1.0$ - basic speed expansion rate of reduction.

$\mathrm{F}=$ positive, then the contour will get reduced.

$\mathrm{F}=$ negative, the outline will shrink.

$\mathrm{F}(\mathrm{K})=-\varepsilon \mathrm{K}-$ curvature velocity and the corresponding speed $\varepsilon$ is related to the smoothness of the contour. If the value of $\varepsilon$ increases, will get a smoother outline.

$G_{\sigma} * I(x, y)$ - convolution of the image, the Gaussian Filter and size of $\sigma$ must be selected according to image position. To complete the curve border, $M$ must have greater value. If the value of $\mathrm{m}$ is too small, the curve will terminate at the edge or a local maxima of the gradient. 


\subsection{K-Means Clustering}

K-means is a clustering algorithm that works on the grouping or classification of the substances by their attributes in $\mathrm{K}$ number where $\mathrm{K}$ is a positive integer (Sarpe, 2010). The algorithm mainly concentrates to minimize the objective function:

Where, $\left\|x_{i}^{(j)}-c_{j}\right\|^{2}$ is the distance measure

$$
J=\sum_{j=1}^{K} \sum_{i=1}\left\|x_{i}^{(j)}-c_{j}\right\|^{2}
$$

$x_{i}^{(j)}$ is data

$c_{j}$ is cluster

The distance measure by Euclidean is given by,

$$
d\left(x_{i}, x_{j}\right)=\left(\sum_{K=1}^{N}\left(x_{i_{K}}-x_{j_{K}}\right)\right)^{1 / 2}
$$

This algorithm undergoes certain steps. They are pointed below:

Fix the number of clusters as K;

Then, randomly select $\mathrm{K}$ pixels representing the initial groups of centroids;

The group which has closer centroid will be assigned with a pixel value;

All the pixels will get allocated only after the centroids are calculated again.

\subsection{Adaptive Thresholding}

Adaptive thresholding is a segmentation technique done on the basis of histogram, which calculates the threshold value without any external assistance. According to the range of histogram multiple thresholds values are fixed(Upadhyay et al., 2016). But only one threshold value is required to separate two regions. In image processing, the images can be represented with pixel values and their intensity. If the level of intensity lies below the threshold value then it has to be assigned again.

Mathematical equation for thresholding is given by,

$$
f(x, y)=\int \begin{gathered}
1, I(x, y)>T(x, y) \\
0, \text { otherwise }
\end{gathered}
$$

Where, $f(x, y)$ is the binary input image $I(x, y)$ and threshold $T(x, y)$ of the image is determined from the histogram values. The pixel above the threshold is set to 1 otherwise 0 .

\section{Image Assessment Parameters}

During the segmentation of the ovarian cyst, there is a chance of merging two cysts as they are smaller in size. In the medical field, it is important to segment the images accurately for proper analysis and early detection of PCOS[8].

Evaluation parameters like Accuracy, Jaccard co-efficient, Precision, Sensitivity (Prabha \& Kumar, 2016) are determined using following expressions,

$$
\begin{aligned}
& \text { Accuracy }=\frac{T P+T N}{(F N+F P+T P+T N)} \\
& \text { Jaccard }=\frac{|A \cap B|}{|A \cup B|} \\
& \text { Precision }=\frac{T P}{(T P+F P)} \\
& \text { Sensitivity }=\frac{T P}{(T P+F N)}
\end{aligned}
$$


where, $\mathrm{A}=$ Ground truth image, $\mathrm{B}=$ Segmented image

$\mathrm{TP}=$ True positive, $\mathrm{TN}=$ True negative, $\mathrm{FP}=$ False positive, $\mathrm{FN}=$ False negative

\section{Importance Of Segmentation Techniques}

As the region of interest and background having similar grey levels in the ultrasound images, it will be a tedious process for the physicians to segment the cyst accurately. If the count of follicles is not calculated correctly then the doctor may misdiagnose the patient. To overcome this issue segmentation has to be done precisely [9-11]. The following figure-1 shows the overall segmentation process.

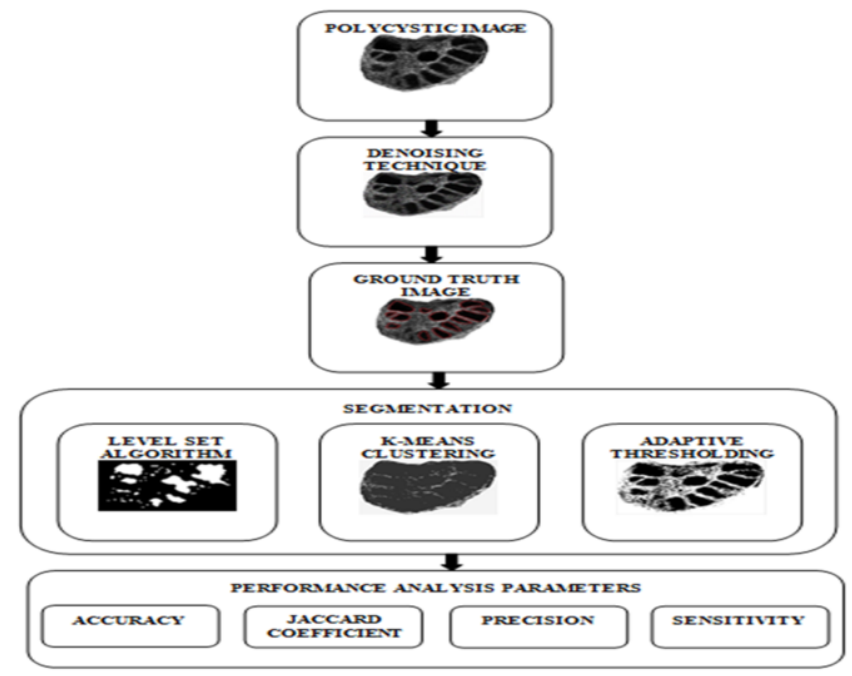

Figure 1. Segmentation Process

\section{Result And Discussion}

The entire image processing work is carried out in Intel Core i3-7020U CPU (a) $2.4 \mathrm{GHz}$ and the simulation results are observed using MATLAB R2019b. The sample image of size $150 \mathrm{X} 200$ pixels is segmented. The performance of various segmentation algorithms are compared quantitatively using Accuracy, Jaccard coefficient, Precision, Sensitivity. The output values obtained for this image are presented in the following Table-1. The plot for the estimation of parameters is shown in Figure-2.

Table 1. Performance analysis of segmentation algorithms

\begin{tabular}{|c|c|c|c|c|}
\hline \multicolumn{5}{|c|}{ SEGMENTATION ANALYSIS } \\
\hline ALGORITHM & ACCURACY & JACCARD & PRECISION & SENSITIVITY \\
\hline ADAPTIVE THRESHOLDING & 0.7084 & 0.7731 & 0.4883 & 0.9796 \\
\hline K-MEANS CLUSTERING & 0.674 & 0.5868 & 0.2665 & 0.9392 \\
\hline LEVEL SET & 0.5377 & 0.2382 & 0.0146 & 0.0099 \\
\hline
\end{tabular}






Figure 2. Comparison of segmentation methods

\section{Conclusion}

In this paper, various segmentation techniques have been analyzed. This paper has been written on the observation of accurate segmentation of ultrasound PCOS images. From this comparative study, it is concluded that the adaptive thresholding method segments the ovarian cyst exactly from the ultrasound images.

\section{References}

[1] Brunda, R., Divyashree, B., \& Shobha Rani, N. (2018). Image segmentation technique- A comparative study. International Journal of Engineering and Technology(UAE), 7(4), 3131-3134. https://doi.org/10.14419/ijet.v7i4.18445

[2] Prabha, D. S., \& Kumar, J. S. (2016). Performance evaluation of image segmentation using objective methods. Indian Journal of Science and Technology, 9(8), 1-8. https://doi.org/10.17485/ijst/2016/v9i8/87907

[3] Qian, S., \& Weng, G. (2016). Medical image segmentation based on FCM and Level Set algorithm. Proceedings of the IEEE International Conference on Software Engineering and Service Sciences, ICSESS, 0(Figure Id), 225-228. https://doi.org/10.1109/ICSESS.2016.7883054

[4] Sarpe, A. I. (2010). Image segmentation with clustering K-means andwatershed transform. 2nd International Conference on Advances in Multimedia, MMEDIA 2010, 1(2), 13-17. https://doi.org/10.1109/MMEDIA.2010.31

[5] Sharma, A., Kumar, R., \& Mansotra, V. (2016). Proposed Stemming Algorithm for Hindi Information Retrieval. International Journal of Innovative Research in Computer and Communication Engineering (An ISO Certified Organization), 3297(6), 11449-11455. https://doi.org/10.15680/IJIRCCE.2016

[6] Singh, L., Singh, S., \& Aggarwal, N. (2019). Proceedings of 2nd International Conference on Communication, Computing and Networking. In 2nd International Conference on Communication, Computing and Networking (Vol. 46). Springer Singapore. https://doi.org/10.1007/978-981-13-1217-5

[7] Singh, T. R., Roy, S., Singh, O. I., Sinam, T., \& Singh, K. M. (2012). A New Local Adaptive Thresholding Technique in Binarization. 8(6), 271-277. http://arxiv.org/abs/1201.5227

[8] Upadhyay, P. K., Chandra, S., \& Sharma, A. (2016). A novel approach of adaptive thresholding for image segmentation on GPU. 2016 4th International Conference on Parallel, Distributed and Grid Computing, PDGC 2016, 652-655. https://doi.org/10.1109/PDGC.2016.7913203.

[9] V.D.Ambeth Kumar, Dr.M.Ramakrishnan, V.D.Ashok Kumar and Dr.S.Malathi (2015) "Performance Improvement using an Automation System for Recognition of Multiple Parametric Features based on Human Footprint. for the International Journal of kuwait journal of science \& engineering, Vol 42, No 1 (2015), pp:109-132.

[10] Ambeth Kumar S. Malathi R. Venkatesan K Ramalakshmi, Weiping Ding, Abhishek Kumar "Exploration of an innovative geometric parameter based on performance enhancement for foot print recognition", Journal of Intelligent and Fuzzy System, vol. 38, no. 2, pp. 2181-2196, 2020.

[11] V.D.Ambeth Kumar, Dr.S.Malathi, V.D.Ashok Kumar (2015) "Performance Improvement Using an Automation System for Segmentation of Multiple Parametric Features Based on Human Footprint” for the Journal of Electrical Engineering \& Technology (JEET), vol. 10, no. 4, pp.1815-1821 , 2015 